\title{
Common Mistakes in Statistical Analysis Reporting: an interview with Amy Frances Moore
}

Amy Frances Moore

Biostatistician, George Washington University, Washington, DC, United States

Email: Amoore20@moorestat.com

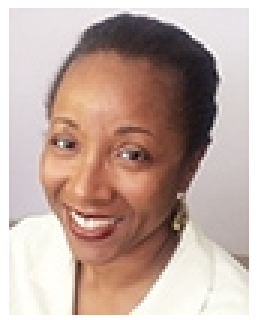

Type of article: Interview

\section{How to cite this article:}

Moore Amy Frances. Common Mistakes in Statistical Analysis Reporting: an interview with Amy Frances Moore. Electron. physician, November 17, 2018,doi: http://dx.doi.org/10.19082/Statstical_Analysis_Interview

\begin{abstract}
Statistical analysis in research requires a well-defined research question, including risk factors and outcome measures. It is also important to report the most appropriate statistical method and results. Amy Frances Moore is a statistics educator and consultant with two Master's degrees in Biostatistics and Public Health Sciences. Ms. Moore is also an application developer who has developed several decision-based tools for the adult-learner. As a statistical-trainer, Ms. Moore has mentored various students in programming and statistics. Ms. Moore has authored two books: "Statistics Workbook for the Non-Techy" and "Statistics for the Math Illiterate" found on Amazon Books. In this interview, Ms. Amy Frances Moore shared her perspective of the common mistakes in statistical analysis reporting from Non-Statisticians.

Keywords: Statistics, Analysis, Mistake, Biostatistics
\end{abstract}

\section{THE INTERVIEW}

What are some challenges as a statistics expert within Biomedical Research?

The nature of biomedical research requires the statistician to be the analyst and the educator. In some instances, teaching others may yield unfavorable results in terms of the analysis. There is a constant conflict between research reporting and statistical validation. There are facilities which need formal training in data handling. In some instances, the facilities lack the personnel and tools for good clinical practice. According to Garcia-Milian, Hershey, and Vukmirovic, who conducted a study on group of Yale-affiliated researchers, researchers lack the time and understanding of the resources required to facilitate statistical analysis (1). There are facilities which lack the presence of a statistician. It can be common place to have a research facility with very little statistical support. As a result, the data samples come to the statistician with deficiencies, such as, inadequate sample size or errors in the data design. Most times the data is salvageable, but there are cases where the absence of a statistician can be quite costly.

What other aspects of biomedical research would facilitate the work of a statistical expert?

Another challenge is the misconception that inferential statistics can be used in every situation. According to Altman, there are cases where the investigator can wrongly assume statistical significance (2). As an expert, it is a challenge to help the researcher accept there is no strong evidence to their hypothesis. Skelly, Dettori, and Brodt provided good examples of the misleading nature of bias in biomedical research (3). I have seen many cases where 
individuals attempted to use convenience sampling with predictive modeling. Suppose you were working at hospital $\mathrm{X}$ and you decide to conduct a study on the patients who come to the clinic at a time point. The sample you collected is a convenience sample, which is not at random. Predictive modeling requires a random sample to ensure there is no bias introduced to the study. Nyirongo, Mukaka, and Kaliani-Phiri outlined the pitfalls of analyzing self-selection for sampling practice (4). As we know any subjectivity in data handling may introduce bias in the results. The researcher must be well-informed with regards to data handling and the danger of introducing bias in their work.

\section{Are there challenges in every sector of biomedical research?}

I have worked in corporate, academia, and the hospital setting with biomedical studies. There are some sectors of research which are regularly monitored by government agencies. In those instances, statistical analysis and reporting must follow strict good clinical practice guidelines. If a pharmaceutical company does not follow the FDA guidelines, the drug may not make it to market. However, other institutions monitor their processes on a more dynamic scale, naturally leading to errors in data handling and analysis. I also note that small academic facilities may not have the resources to follow good clinical practice guidelines in detail. Large facilities may have more researchers than actual experts on statistics. It is dependent upon the working environment.

\section{In your experience, which sector of biomedical research needs the most support?}

There is no sector of biomedical research which needs special attention. I rather see the emphasis being put on ensuring our research publications contain sound evidence of statistics with the proper handling of data. And most of all, each researcher should have access to data tools which facilitate accurate statistics and reporting.

What skills should every biomedical researcher possess in order to be successful in their statistical reporting? A biomedical researcher should know the statistical assumptions that must be met to analyze their data. I have seen many cases where the data design did not agree with the statistical method of choice. I have seen cases where the mean statistic is not reported with a standard deviation. Another example, investigator often confuse the pairedsamples t-test for the independent-samples t-test. Or the researcher may attempt to conduct an Independent-samples t-test with some of the same participants in both groups. Bajwa attempted to make anesthesiologists aware of importance of proper study design and implementation (5). The article gives a brief tutorial of the steps which must occur before study initiation. The overall message is clear, there are specific tasks that must occur in order to ensure the integrity of your statistical analysis.

\section{Are there any statistics trainings that you recommend for biomedical researchers?}

My practical answer is to see a statistics expert before you collect your data. If you can not find an expert, I recommend training in the assumptions for parametric and non-parametric statistics. I have trained others to recognize which data structure is appropriate for a given statistical model. I feel that more scientists should invest in learning proper statistical techniques. It will avoid mistakes later in a study.

Ms. Amy Frances Moore is a statistics educator and consultant with two Master's degrees in Biostatistics and Public Health Sciences. Ms. Moore is also an application developer who has developed several decision-based tools for the adult-learner. As a statistical-trainer, Ms. Moore has mentored various students in programming and statistics. Ms. Moore has authored two books: "Statistics Workbook for the Non-Techy" and "Statistics for the Math Illiterate"

\section{References:}

1) Garcia-Millian R, Hersey D, Vukmirovic M, Duprilot F. Data challenges of biomedical researchers in the age of omics. PeerJ. 2018; 6: e5553. doi: 10.7717/peerj.5553. PMID: 30221093, PMCID: PMC6138043.

2) Altman DG. The scandal of poor medical research. BMJ. 1994; 308(6924): 283-4. doi: 10.1136/bmj.308.6924.283. PMID: 8124111, PMCID: PMC2539276.

3) Skelly AC, Dettori JR, Brodt ED. Assessing bias: the importance of considering confounding. Evid Based Spine Care J. 2012; 3(1): 9-12. doi: 10.1055/s-0031-1298595. PMID: 23236300, PMCID: PMC3503514.

4) Nyirongo VB, Mukaka MM, Kalilani-Phiri LV. Statistical Pitfalls in Medical Research. Malawi Med J. 2008; 20(1): 15-8. PMID: 19260441, PMCID: PMC3345655.

5) Bajwa SJ. Basics, common errors and essentials of statistical tools and techniques in anesthesiology research J Anaesthesiol Clin Pharmacol. 2015; 31(4): 547-53. doi: 10.4103/0970-9185.169087. PMID: 26702217, PMCID: PMC4676249. 